\title{
DISTRIBUIÇÃO E BIOMASSA DE MACROALGAS EM UM MANGUEZAL DA BAÍA DA BABITONGA, SC: RESULTADOS PRELIMINARES
}

\author{
CUNHA,S.R. 1,2; NASCIMENTO,J.1,3; LIMA,G.B. '; ZACHARJASIEWICZ,G. ${ }^{1}$; \\ CRESTANI,D.E.V.'; MAFRA Jr.,L.L. ; PAZETO,F.D.'; SANT'ANNA,F.1 \& C.S.B. COSTA ${ }^{2}$ \\ ${ }^{1}$ Centro de Ciências Tecnológicas, da Terra e do Mar - Universidade do Vale do Itajaí \\ (CTTMar/UNIVALI) Rua Uruguai, 458 - Cx.Postal 360, Cep: 88.302-202 - Itajaí, SC. e- \\ mail:srcunha@univali.rct-sc.br \\ 2 Universidade do Rio Grande, Lab. Comunidades Vegetais Costeiras. (FURG) Cx. \\ Postal 474 - CEP: 96201-900 Rio Grande, RS. \\ ${ }^{3}$ Bolsista Probic-UNIVALI
}

\begin{abstract}
RESUMO
Este trabalho tem como objetivo determinar a distribuição e quantificar a biomassa de macroalgas presentes nas raízes e troncos das árvores de manguezal da Baía de Babitonga. Para tal, as macroalgas foram coletadas mensalmente (setembro/1997 a junho/1998) em 3 níveis topográficos dentro do manguezal (Linhas A, B e C). Em cada linha a coleta foi estratificada de acordo com a altura das algas no tronco ( 0 a $10 \mathrm{~cm}$ de altura, $10 \mathrm{a} 20 \mathrm{~cm}$, etc). As espécies de Rhodophyta observadas, em ordem decrescente de biomassa, foram: Bostrychia calliptera, Bostrychia pinnata, Bostrychia radicans f. radicans, Bostrychia montagnei, Catenella caespitosa, Caloglossa leprieurii, Bostrychia tenella, Caloglossa ogazawaraensis, Bostrychia radicans f. moniliforme, Bostrychia moritziana, Polysiphonia howei, Bostrychia binderi, Gelidium spp., Polysiphonia tepida. As Chlorophyta são Bloodleopsis pusilla, Cladophoropsis membranacea, Rhizoclonium spp., Ulvaria oxysperma, Enteromorpha spp. Há uma forte variação horizontal e vertical na biomassa e na contribuição de cada espécie para a biomassa total. Na franja do manguezal (linha A) as algas ocupam uma altura

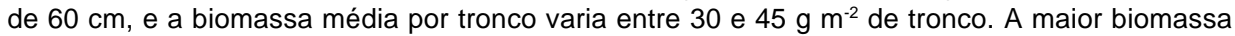
ocorre entre 10 e $30 \mathrm{~cm}$, chegando a $75 \mathrm{~g} \mathrm{~m}^{-2}$ de tronco. Na porção intermediária (linha B) as algas ocupam uma altura de $30 \mathrm{~cm}$ e a biomassa média varia entre 20 e $30 \mathrm{~g} \mathrm{~m}^{-2}$ de tronco. A biomassa é

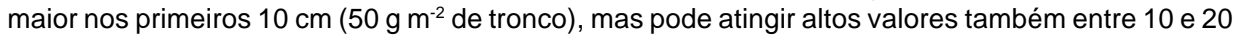
$\mathrm{cm}$. Na porção interna do manguezal (linha C) as algas ocupam uma altura de $20 \mathrm{~cm}$, e a biomassa média varia entre $10 \mathrm{e} 20 \mathrm{~g} \mathrm{~m}^{-2}$ de tronco. Assim como na linha $\mathrm{B}$, a biomassa é maior nos primeiros $10 \mathrm{~cm}\left(35 \mathrm{~g} \mathrm{~m}^{-2}\right.$ de tronco), mas pode atingir altos valores também entre 10 e $20 \mathrm{~cm}$. As algas verdes Bloodleopsis pusilla, Cladophoropsis membranacea, Rhizoclonium, e as algas vermelhas Bostrychia montagnei, Caloglossa leprieurii e Caloglossa ogazawaraensis estão presentes principalmente nos primeiros $10 \mathrm{~cm}$ de altura, raramente ocorrendo acima de $20 \mathrm{~cm}$. Bostrychia radicans f. radicans é a alga mais amplamente distribuída, com maior freqüência de ocorrência e a mais abundante na região superior do tronco nas três linhas. As algas que apresentam a maior biomassa, especialmente na linha A, são Bostrychia calliptera e Bostrychia pinnata, que também apresentam distribuição vertical e horizontal ampla, mas são mais abundantes entre 10 e $30 \mathrm{~cm}$ de altura. Os resultados obtidos até o momento indicam que as macroalgas podem ser vistas como um componente produtor representativo dentro do manguezal. Entretanto, para uma avaliação da biomassa de macroalgas no manguezal como um todo, é necessário quantificar a biomassa de algas em pneumatóforos. Para avaliar a contribuição destas algas para a produção primária do manguezal, os dados de biomassa devem ser complementados com experimentos de crescimento e produtividade, com relação aos fatores ambientais.
\end{abstract}

Palavras chave: macroalgas, manguezal, produção primária, Bostrychia, Rhodophyta 


\title{
SEAWEED DISTRIBUTION AND BIOMASS IN A MANGROVE OF BAÍA DA BABITONGA, SC: PRELIMINARY RESULTS
}

\begin{abstract}
This work aims to assess distribution and biomass of epiphyte macroalgae of mangrove roots in the Babitonga Bay. The macroalgae were collected monthly in three topographyc levels into the mangrove (Places A, B and C). In each place the collect was stratified in the trees (0 to $10 \mathrm{~cm}$ high, 10 to $20 \mathrm{~cm}$, etc). The observed Rhodophyta, from higher to smaller biomass, were: Bostrychia calliptera, Bostrychia pinnata, Bostrychia radicans f. radicans, Bostrychia montagnei, Catenella caespitosa, Caloglossa leprieurii, Bostrychia tenella, Caloglossa ogazawaraensis, Bostrychia radicans $\mathrm{f}$. moniliforme, Bostrychia moritziana, Polysiphonia howei, Bostrychia binderi, Gelidium spp., Polysiphonia tepida. The observed Chlorophyta were: Bloodleopsis pusilla, Cladophoropsis membranacea, Rhizoclonium spp., Ulvaria oxysperma, Enteromorpha spp. There are strong variation on horizontal and vertical biomass and on contribution of each species to total biomass. In the mangrove fringe (place $A$ ) the algae occur from 0 to $60 \mathrm{~cm}$ high in the trees, the mean biomass along this high varies 30 and $45 \mathrm{~g} \mathrm{~m}^{-2}$ of substrate. Higher values of biomass occur between 10 and $30 \mathrm{~cm}$ high, rising to $75 \mathrm{~g} \mathrm{~m}^{-2}$. On place $\mathrm{B}$ (intermediate tidal flooding) the algae occur from 0 to $30 \mathrm{~cm}$ high in the trees, the mean biomass along this high varies 20 and $30 \mathrm{~g} \mathrm{~m}^{-2}$ of substrate. Higher values of biomass occur between 0 and $10 \mathrm{~cm}$ high ( $50 \mathrm{~g} \mathrm{~m}^{-2}$ of substrate), but high values may also occur between 10 and 20 $\mathrm{cm}$ high. In the inner mangrove (place $\mathrm{C}$ ) the algae occur from 0 to $20 \mathrm{~cm}$ high in the trees, the mean biomass along this high varies 10 and $20 \mathrm{~g} \mathrm{~m}^{-2}$ of substrate. Like place $\mathrm{B}$, higher values of biomass occur between 0 and $10 \mathrm{~cm}$ high ( $35 \mathrm{~g} \mathrm{~m}^{-2}$ of substrate), but high values may also occur between 10 and $20 \mathrm{~cm}$ high. The green algae, Bloodleopsis pusilla, Cladophoropsis membranacea, Rhizoclonium, and the red algae, Bostrychia montagnei, Caloglossa leprieurii and Caloglossa ogazawaraensis were mainly present from 0 to $10 \mathrm{~cm}$ high, and rarely occur above $20 \mathrm{~cm}$. Bostrychia radicans f. radicans presents the most broad distribution, has the higher frequency of occurrence in all places and all strata, and it is the main algae in the upper strata. The higher values of biomass, specially on place A, are due to Bostrychia calliptera and Bostrychia pinnata. This two algae also have a broad vertical and horizontal distribution, but they have higher values from 10 to $30 \mathrm{~cm}$ high. The results found until now suggest that macroalgae may be an important component of mangrove primary production. Meanwhile, to evaluate the mangrove magroalgae biomass, we need to include algae epiphytic on pneumatophores. And to evaluate the contribution of these algae to the total primary production of the mangrove, it will be necessary to complement biomass data with ecophysiological experiments, including the responses of growth and photosynthetic rates to environmental factors.
\end{abstract}

Key Words: macroalgae, seaweed, algae, mangrove, primary production, Bostrychia, Rhodophyta

\section{INTRODUÇÃO}

Os manguezais ocorrem ao longo de linhas de costa abrigadas tropicais e subtropicais, e têm sido considerados como sistemas altamente produtivos (Mann, 1982, Day et al., 1989). Estes ecossistemas podem ser vistos como uma interface entre os ecossistemas terrestres e marinhos, fornecendo vias de transporte e transformação de materiais entre ambos (Brown, 1984).

Devido a sua elevada produtividade (entre 200 e $1000 \mathrm{~g} \mathrm{C} \mathrm{m}^{-2}$ ano-1 $^{-1}$ ), acredita-se que o funcionamento natural dos manguezais pode sustentar uma alta produtividade secun- dária, na forma de pescarias estuarinas e costeiras (Cintron \& Schaeffer-Novelli, 1983; Turner, 1985; Turner \& Boesch, 1988), pois além de prover fontes alimentares, os manguezais oferecem habitats de proteção e reprodução para organismos estuarinos e marinhos, muitos dos quais com grande interesse econômico. Entre estes se destacam a ostra (Crassostrea spp.), o sururu (Mytella spp.), o berbigão (Anomalocardia brasiliana), os caranguejos guaiamú e uçá (Cardisoma guanhumi e Ucides cordatus, respectivamente), o camarão (Penaeus sp.), os peixes corvina (Micropogonias furnieri), robalo (Centropomus sp) e tainha (Mugil spp.), entre outros. 
As estimativas de produtividade dos manguezais (Flores-Verdugo, et al., 1987; Amarasinghe \& Balasubramaniam, 1992; Tovilla-Hernandez \& Gonzales-Angelito, 1994; Lee, 1995; Mackey \& Smail, 1995) geralmente levam em conta apenas a produção de serrapilheira (material vegetal reprodutivo e vegetativo que cai das árvores devido a senescência, estresse e/ou forças mecânicas dos ventos e das chuvas; Brown 1984). Entretanto, outros produtores primários estão presentes no manguezal: as porções de troncos e raízes das árvores sujeitas a inundação durante a maré alta, encontram-se recobertas por comunidades de macroalgas, comumente denominadas "Bostrychietum" (sensu Post,1968), compostas por espécies dos gêneros Bostrychia, Caloglossa, Catenella, Cladophoropsis, Rhizoclonium e Bloodleopsis, entre outros (Oliveira, 1984; Hadlich, 1984; Hadlich \& Bouzon, 1985; Cordeiro Marino et al., 1992; Shirata, 1993).

As algas estão entre os mais eficientes conversores de energia solar em energia química utilizável (Vonshak \& Maske, 1980). Estudos recentes tem demonstrado que as macroalgas representam uma fonte significativa de produção primária em alguns ecossistemas de manguezais, constituindo importantes fontes de energia e elementos minerais para as redes tróficas estuarinolagunares (Rodriguez \& Stoner, 1990; Steinke \& Naidoo, 1990; Karsten et al., 1994; Narasimha Rao, 1995). As macroalgas, que podem ser utilizadas diretamente através da pastagem por diversos organismos (Ambler et al., 1993), apresentam maior teor de nitrogênio e maior palatibilidade quando comparadas a vegetais superiores (Tenore, 1977). Além da sua contribuição direta para a teia trófica estuarina, as algas apresentam taxas de decomposição bastante elevadas, liberando nutrientes orgânicos e inorgânicos e carbono orgânico dissolvido e particulado, que podem ser utilizados localmente ou ser exportados para águas adjacentes (Hanisak, 1993).
Estimativas de produção de macroalgas de manguezal realizadas por Steinke \& Naidoo (1990) mostram que a biomassa de macroalgas em pneumatóforos pode atingir até $5 \mathrm{~g} \mathrm{PS} \mathrm{m}^{-2}$ de manguezal, gerando uma produção anual de detritos de $220 \mathrm{~g} \mathrm{PS} \mathrm{m}^{-2}$ ano $^{-1}$. Em análises realizadas por Rodriguez \& Stoner (1990) no estuário de Laguna Joyuda (Porto Rico) foram observados valores de biomassa das comunidades algais associadas às raízes de Rhizophora mangle, similares aos valores anuais de produção de serrapilheira, o que justificaria a dominância de cadeias alimentares algais naquele estuário.

Apesar do crescente interesse sobre as macroalgas de manguezal e sua contribuição para o ecossistema, estudos quantitativos que permitam avaliar a contribuição das macroalgas para a produção primária do manguezal, são bastante demorados, envolvendo vários aspectos biológicos e ecofisiológicos destas macroalgas. Entre os estudos realizados na costa brasileira destacam-se os qualitativos, que são de grande importância para o embasamento de estudos qualitativos posteriores. Joly $(1954,1957)$ referiu-se a algumas espécies de macroalgas para manguezais de Santos (SP). Oliveira (1969) listou as espécies presentes em manguezais do Espírito Santo, fazendo algumas considerações ecológicas sobre as mesmas. Michell et al. (1974) mostraram que, em manguezais da Baía de Guanabara (RJ), as associações que crescem sobre pneumatóforos são diferentes daquelas que crescem sobre tronco e raízes de escora das árvores de manguezal. Pedrini (1980) listou as espécies presentes em manguezais da Baía de Sepetiba (RJ), reconhecendo 11 espécies exclusivas destes ambientes. Camargo (1982), em experimentos com substratos artificiais em manguezais na região de Cananéia, observou que a colonização por macroalgas é lenta naquela área. Oliveira (1984) enfatizou a importância de estudos sobre estas macroalgas, fazendo algumas 
considerações ecológicas. Miranda (1986) descreveu a composição, fenologia e distribuição de macroalgas em manguezais do Rio Ceará. Paula et al. (1989) mostraram a ocorrência de várias espécies em manguezais da Ilha de Maracá (AM). Braga et al. (1990) e Eston et al. (1991) estudaram padrões espaciais e temporais de macroalgas em manguezais da região de Cananéia. Eston et al. (1992) estudaram padrões de colonização de substratos artificiais na mesma área. Cordeiro-Marino et al. (1992) fizeram uma revisão sobre o atual conhecimento sobre as macroalgas de manguezal na América Latina. Shirata (1993) listou as espécies de macroalgas nos manguezais da Baía de Guaraqueçaba (PR) e estudou seus padrões sazonais.

Em Santa Catarina destacam-se o levantamento de algas Rhodophyta bentônicas ao longo da costa realizado por Cordeiro Marino (1978), que cita algumas espécies presentes em manguezais, e os levantamentos de Hadlich (1984) e Hadlich \& Bouzon (1985), que estudaram, respectivamente, os padrões sazonais das Chlorophyta e das Rhodophyta do manguezal de Itacorubi (Florianópolis).

A caracterização das comunidades de macroalgas presentes nos manguezais da Baía de Babitonga e a avaliação de sua biomassa representa o primeiro passo para a avaliação da contribuição destas algas para a produção primária destes manguezais. Este trabalho tem como objetivo avaliar a distribuição e quantificar a biomassa das macroalgas de manguezal presentes na porção média da Baía de Babitonga.

\section{Área de estudo}

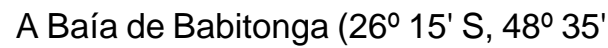
W, figura 1) representa a principal área estuarina do Estado de Santa Catarina, com um corpo d'água de aproximadamente 167 $\mathrm{Km}^{2}$. Esta baía possui uma extensa formação de manguezais, que ocupam uma área de aproximadamente $60 \mathrm{Km}^{2}$ (Herz, 1991), o que representa em torno de $75 \%$ dos manguezais do estado.

Os manguezais apresentam bosques de Laguncularia racemosa, Avicennia schaueriana e Rhizophora mangle, com ocorrência de Acrostichum aureum e Hibiscus pernanbucencis na zona de limite com a restinga. A ocorrência de Spartina alterniflora em bancos de maré ou formando franjas frontais ao manguezal é bastante comum em toda a baia. No Rio Palmital, desde sua região mediana até a mais interna, ocorre com elevada abundância "marismas" de Crinum sp, a açucena-da-água (Cunha et al., 1997).

O clima na região é mesotérmico úmido, com verão quente e sem estação seca. $O$ teor de umidade do ar é sempre elevado, variando entre 68 e $89 \%$. A precipitação mensal varia entre $402 \mathrm{~mm}$ (janeiro) e $106 \mathrm{~mm}$ (agosto e a temperatura média mensal varia entre 16.5 e $25.6^{\circ} \mathrm{C}$ ) (figura 2A; Climerh/ Epagri). A baía recebe contribuições das bacias hidrográficas dos rios Palmital, Pirabeiraba, Cachoeira, Três Barras e Piraí (SEPLAN-SC, 1988).

O manguezal estudado localiza-se na porção média da Baía de Babitonga (figura 1, estação 3), que apresenta características intermediárias de estrutura de bosque, salinidade e hidrodinâmica, entre a porção mais interna e a desembocadura da baía, segundo estudos preliminares na região (Cunha et al.,1997).

\section{METODOLOGIA}

A coleta das macroalgas foi efetuada segundo metodologia proposta por Tsuda e Abbott (1985) e Alveal \& Romo (1995). As algas presentes sobre as raízes de ancoragem e troncos das árvores de manguezal foram coletadas através da raspagem da superfície de ocorrência, utilizando-se amostradores de $25 \mathrm{~cm}^{2}(2,5 \mathrm{~cm}$ de largura por $10 \mathrm{~cm}$ de altura), ao longo do comprimento da raiz, segun- 


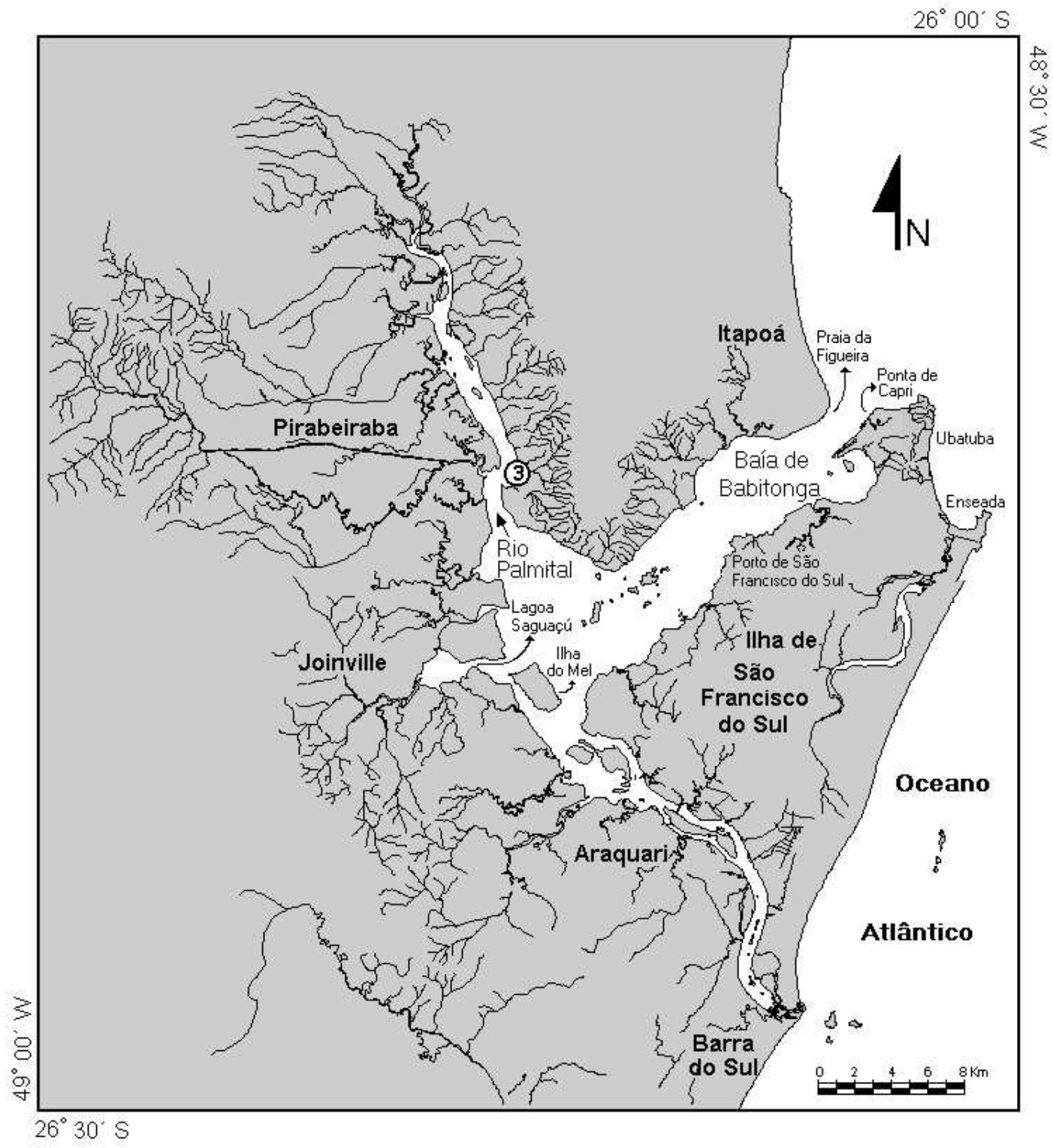

Figura 1: Baía de Babitonga, SC, mostrando a bacia hidrográfica e estação de coleta de macroalgas e dados abióticos.

do a estratificação devido ao nível de inundação $(0$ a $10 \mathrm{~cm}$ do sedimento, 10 a $20 \mathrm{~cm}$, e assim por diante).

Para os testes amostrais foram coletadas 20 réplicas de cada tipo de amostra, na parcela 1 da estação 3. Nas amostragens subsequentes foram coletadas 5 réplicas de cada tipo de amostra, de acordo com os resultados dos testes amostrais. A amostragem ocorreu na franja, próximo à margem do manguezal (Linha $A)$, na porção intermediária (Linha B) e na porção mais in- 
terna, próximo à restinga (Linha C). Cada Linha de amostragem consiste em uma área de $1000 \mathrm{~m}^{2}$ (100 m paraletamente à margem por $10 \mathrm{~m}$ perpendicularmente à margem). A posição das amostras dentro de cada linha foi determinada com o auxílio de uma tabela de números aleatórios.

Foi realizada uma coleta isolada de macroalgas em setembro de 1997, e a partir de dezembro de 1997 a coleta foi realizada mensalmente.

As macroalgas coletadas foram acondicionadas em sacos plásticos e conduzidas ao laboratório, onde as amostras foram mantidas sob refrigeração. Durante o processamento o material foi lavado, separado por espécie em microscópio estereoscópico, seco em estufa a $60^{\circ}$ até atingir peso constante e pesado em balança analítica.

A identificação das algas foi realizada com base na bibliografia especializada (Cordeiro-Marino, 1978; Hadlich, 1984; Hadlich \& Bouzon; 1985; King \& Puttock, 1989). As algas vermelhas foram separadas por espécie. Algumas espécies de um mesmo gênero que apresentaram quantidades indetectáveis quando separadas foram agrupadas por gênero. As algas verdes, que são de difícil separação, após identificadas foram pesadas conjuntamente. A alga verde Rhizoclonium foi mantida a nível de gênero. As algas Cyanophyta não foram identificadas. A biomassa foi expressa em $\mathrm{g} \mathrm{PS} \mathrm{m}^{-2}$ de tronco (gramas de peso seco de algas por metro quadrado de tronco).

Dados de temperatura do ar e da água, salinidade, transparência e profundidade da água foram coletados diariamente, sempre num mesmo horário (11:00 h) durante o período amostral, a uma distância de 500 m da estação de amostragem (estação 3). Esta coleta foi efetuada por um morador local treinado. Dados complementares de salinidade da água foram coletados a cada amostragem de macroalgas.

\section{RESULTADOS E DISCUSSÃO}

Dados abióticos

As variáveis abióticas medidas diariamente por um morador local apresentaram padrões coerentes e compatíveis entre si e com dados coletados nos dias de amostragem. A temperatura diária do ar variou entre 15 e $33^{\circ} \mathrm{C}$, e a temperatura da água entre 15 e $30^{\circ} \mathrm{C}$ (figura $2 \mathrm{~B}$ ). Os dados diários de salinidade apresentam uma variação sazonal fortemente definida, com valores mais baixos durante o verão (figura $2 \mathrm{C}$ ), período de maior precipitação pluviométrica na Baía de Babitonga. Os valores de salinidade variaram de 0 a 25, e são compatíveis com os valores observados durante as amostragens de serrapilheira (figura 2C). A maré é semidiurna e apresenta uma variação média de 1,8 m. A transparência da água é relativamente baixa devido ao aporte de material em suspensão da bacia hidrográfica (figura 2D). Entretanto, durante o período de baixa precipitação, a transparência pode atingir quase $100 \%$ da coluna d'água (figura 2D).

\section{Distribuição e biomassa das macroalgas no manguezal}

Foram observadas associadas aos manguezais da Baía de Babitonga várias espécies de Rhodophyta, algumas de Chlorophyta e algumas de Cyanophyta, sendo que estas últimas não foram identificadas. Espécies de Phaeophyta não ocorreram nas amostragens, nem foram observadas em amostragens qualitativas complementares. As Rhodophyta observadas até o momento são: Bostrychia binderi, Bostrychia calliptera, Bostrychia montagnei, Bostrychia moritziana, Bostrychia pinnata, Bostrychia radicans $\mathrm{f}$. moniliforme, Bostrychia radicans f. radicans, Bostrychia tenella, Caloglossa leprieurii, Caloglossa ogazawaraensis, Catenella caespitosa, e em menor abundância, Gelidium spp., Polysiphonia howei, Polysiphonia tepida. 

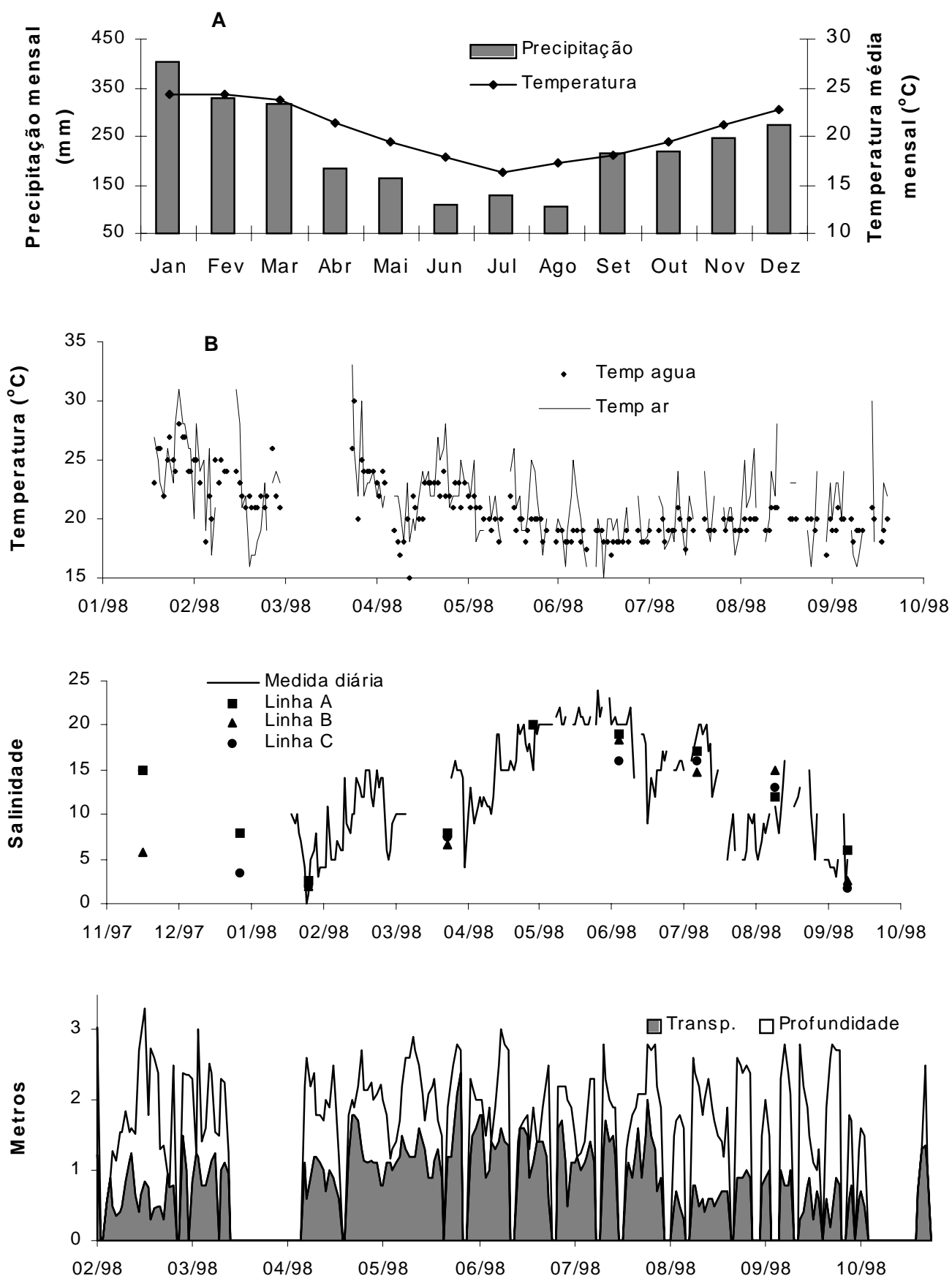

Figura 2: Dados abióticos. A: Dados históricos de precipitação e temperatura (Climerh/Epagri) para a região da Baía de Babitonga; B: Temperatura da água e do ar, coletadas diariamente próximo à estação de coleta de algas; C: Salinidade da água, coletada diáriamente e dentro do manguezal, durante as coletas de macroalgas (linhas A, B e C); D: profundidade da coluna d'água e transparência percentual, coletada diariamente. 
As Chlorophyta são Bloodleopsis pusilla, Cladophoropsis membranacea, Rhizoclonium spp., e em menor abundância, Ulvaria oxysperma, Enteromorpha spp. Ocorrem também com elevada freqüência, vários gêneros de Cyanophyta.

Problemas taxonômicos foram encontrados com relação às espécies Bostrychia calliptera e Bostrychia pinnata. A espécie identificada como Bostrychia calliptera, com base em Cordeiro-Manirno (1978), começou a mostrar variações morfológicas no tamanho e no tipo de corticação do eixo principal. A partir da observação destas diferenças morfológicas, procurou-se literatura mais atualizada para avaliar se isto poderia ser uma variação morfológica dentro da espécie Bostrychia calliptera, como ocorre em Bostrychia radicans (Bostrychia radicans $f$. radicans, $B$. radicans $f$. moniliforme, entre outras).

Aparentemente, a espécie referida por Cordeiro-Manirno (1978) e por outros pesquisadores brasileiros como Bostrychia calliptera seria, de acordo com King \& Puttock (1989), classificada como Bostrychia pinnata. A alga classificada como Bostrychia calliptera apresenta corticação bem desenvolvida no eixo principal, enquanto Bostrychia pinnata apresenta corticação apenas na base do eixo principal (estipe). Uma característica complementar na separação das duas espécies é o comprimento dos ramos. Os ramos de crescimento indeterminado e determinado medem, respectivamente, 20-40 mm e 2-3 mm em Bostrychia pinnata e 20-60 mm e 3-5 mm em Bostrychia calliptera.

A observação sistemática destas duas morfologias e a separação das mesmas em frações diferenciadas dentro de uma mesma amostra iniciou-se em fevereiro de 1998. Nas amostras analisadas anteriormente, todos os exemplares foram considerados como Bostrychia calliptera. Devido a isto, os dados referentes a Bostrychia calliptera nos meses de setembro/97, dezembro/97 e janeiro/98 podem ser considerados como o somatório de biomassa de Bostrychia calliptera e Bostrychia pinnata.

Há uma considerável variação espacial na biomassa e na composição da comunidade. Devido ao perfil topográfico do manguezal, na linha $A$ as algas podem ocupar até $60 \mathrm{~cm}$ de altura do tronco das árvores, enquanto na linha $B$ podem ocupar até $30 \mathrm{~cm}$ e na linha $C$ até $20 \mathrm{~cm}$ (figura $3 A$ ). Com relação à altura no tronco, nas linhas $\mathrm{B} e \mathrm{C}, \mathrm{a}$ maior biomassa ocorre entre 0 e $10 \mathrm{~cm}$ de altura. Devido à maior freqüência de alagamento na linha $A$, a biomassa média de algas nos troncos nesta linha é superior às demais (figura 3A). Na linha A, apesar da biomassa nos primeiros $10 \mathrm{~cm}$ ser relativamente similar à biomassa das linhas $\mathrm{B}$ e $\mathrm{C}$, os maiores valores são observados entre 10 e $30 \mathrm{~cm}$ de altura. Acima dos $30 \mathrm{~cm}$ a biomassa volta a cair (figura $3 \mathrm{~A}$ ).

$\mathrm{Na}$ linha $\mathrm{A}$, a biomassa média por tronco (ao longo dos $60 \mathrm{~cm}$ de altura colonizados por algas) não apresenta padrão sazonal definido, com valores variando entre 29 e $45 \mathrm{~g}$ $\mathrm{m}^{-2}$ e máximo em maio/98 (figura 3B). Na linha $B$ a biomassa média por tronco (ao longo dos $30 \mathrm{~cm}$ de altura) varia entre 15 e $45 \mathrm{~g} \mathrm{~m}^{-}$ ${ }^{2}$, apresentando o máximo em março/98 (figura $3 \mathrm{C}$ ). Na linha $\mathrm{C}$ a biomassa média por tronco (ao longo dos $20 \mathrm{~cm}$ de altura) varia entre 9 e $33 \mathrm{~g} \mathrm{~m}^{-2}$, apresentando o máximo em fevereiro/98 (figura 3D).

A maior parte das espécies está presente em todas as alturas de tronco e nas três linhas de amostragem (figura 4). Entretanto, a contribuição de cada espécie varia muito com a altura no tronco e com a posição dentro do manguezal, refletindo a tolerância de cada espécie à dessecação e ao aporte de sedimentos.

Nos primeiros $10 \mathrm{~cm}$ de altura praticamente todas as espécies encontradas neste estudo estão presentes. É nesta faixa que as algas verdes, Bloodleopsis pusilla e Cladophoropsis membranacea, ocorrem em maior freqüência e biomassa. A contribuição destas algas para os primeiros $10 \mathrm{~cm}$ da li- 

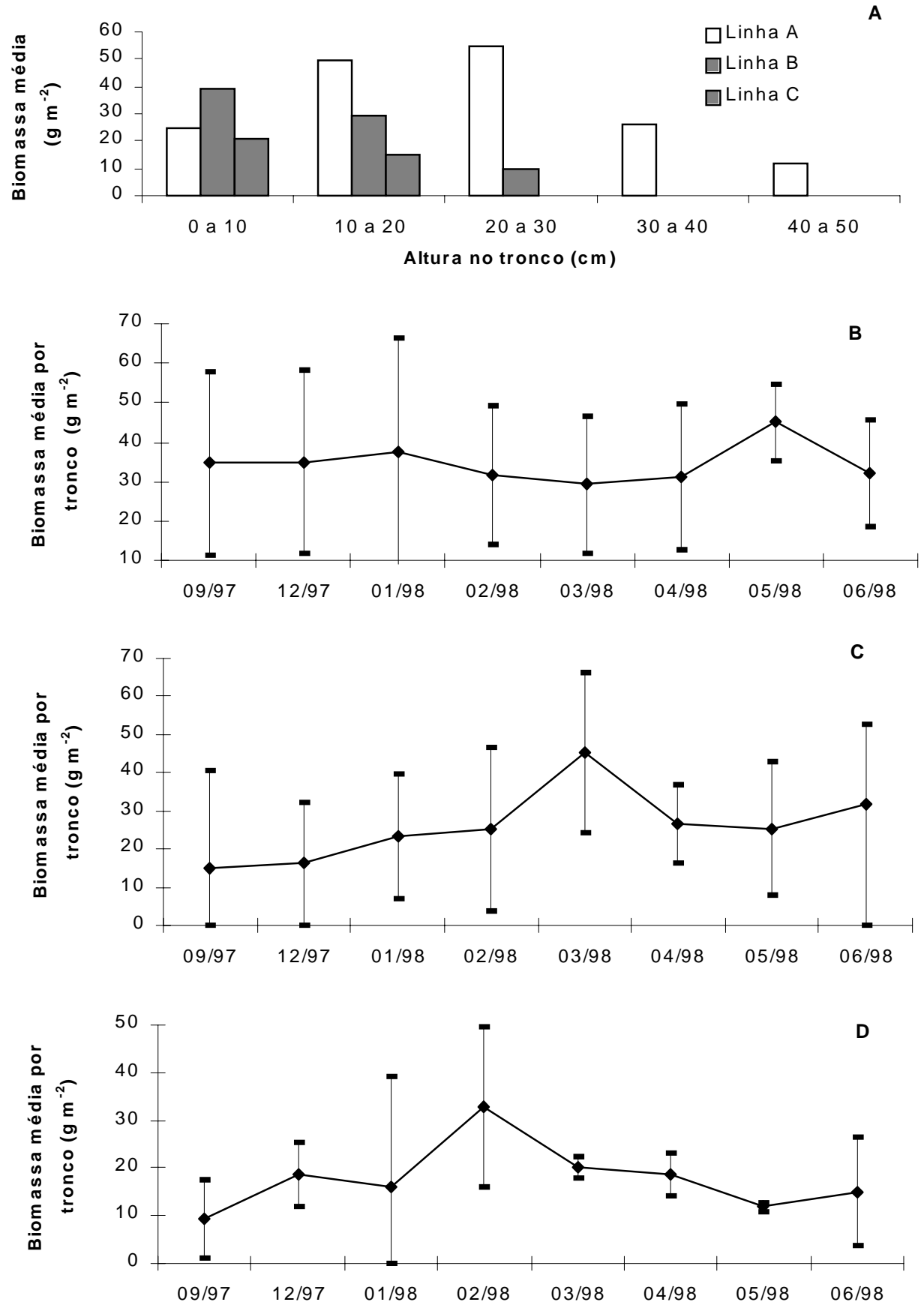

Figura 3: A: Biomassa média (média \pm 1 desvio padrão) das algas para todo o período amostrado, considerando a linha e a altura no tronco, com relação ao sedimento; B, C e D: Variação sazonal da biomassa média para todas as alturas de tronco, respectivamente para as linhas A (de 0 a $60 \mathrm{~cm}), B(0$ a $30 \mathrm{~cm})$ e C $(0$ a $20 \mathrm{~cm})$. 
nha $A$ pode variar entre 5 e $65 \%$ da biomassa média. Na linha B podem contribuir com 15 a $90 \%$ da biomassa média, e na linha $C$ podem contribuir com 75 a $100 \%$ da biomassa média (figura 4). Entre 10 e $20 \mathrm{~cm}$ a contribuição das algas verdes varia entre 0 e $5 \%$ da biomassa média, tanto na linha $A$ como na linha $B$ (figuras 4A e 4B). Na linha $C$ entre 10 e $20 \mathrm{~cm}$, a contribuição das verdes varia entre 0 e $35 \%$ (figura $4 C$ ). Acima de $20 \mathrm{~cm}$, a única alga verde que aparece é Rhizoclonium. Embora esta seja a única alga verde presente acima dos $20 \mathrm{~cm}$, este gênero é amplamente distribuído no manguezal, ocorrendo em todas as linhas e em todas as alturas, sempre associado às algas vermelhas, mas sempre com valores de biomassa muito baixos, geralmente indetectáveis. Este gênero ocorre com maior freqüência na linha $A$ e entre 10 e $30 \mathrm{~cm}$ de altura.

A alga mais amplamente distribuída (maior freqüência de ocorrência em todas as linhas e alturas) é Bostrychia radicans $\mathrm{f}$. radicans (figura 4). A maior contribuição desta alga para a biomassa média ocorre nas áreas sujeitas a menor freqüência de alagamento, podendo atingir até $100 \%$ da biomassa acima de $40 \mathrm{~cm}$ na linha $A$ e acima de $10 \mathrm{~cm}$ na linha $C$. Na linha $B$, esta espécie contribui com 20 a $85 \%$ da biomassa acima de $10 \mathrm{~cm}$. O maior valor de biomassa observado para Bostrychia radicans $\mathrm{f}$. radicans foi de $23,9 \mathrm{~g} \mathrm{~m}^{-2}$ na linha $A$, na linha $B$ foi de 22,7 $\mathrm{g} \mathrm{m}^{-2}$ e na linha $C$ foi de $42,7 \mathrm{~g} \mathrm{~m}^{-2}$.

Em termos de biomassa Bostrychia calliptera apresenta os maiores valores observados para a linha $A\left(60,9 \mathrm{~g} \mathrm{~m}^{-2}\right)$, seguida por Bostrychia pinnata $\left(45,2 \mathrm{~g} \mathrm{~m}^{-2}\right)$ e por Bostrychia radicans $\mathrm{f}$. radicans $\left(23,9 \mathrm{~g} \mathrm{~m}^{-2}\right)$. Acima dos $10 \mathrm{~cm}$, estas três espécies representam de 60 a $100 \%$ da biomassa na linha A (figura 4A). Na linha B a dominância destas três espécies se repete acima de $10 \mathrm{~cm}$. Entretanto, nos primeiros $10 \mathrm{~cm}$ desta linha, Bostrychia montagnei cresce em importância, sendo a alga vermelha mais representativa em termos de biomassa, atingindo valores de até $32,4 \mathrm{~g} \mathrm{~m}^{-2}$ (figura 4B). Na linha C, as algas verdes entre 0 e $10 \mathrm{~cm}$ de altura e a alga vermelha Bostrychia radicans $f$. radicans entre 10 e $20 \mathrm{~cm}$ de altura, representam quase a totalidade da biomassa observada (figura $4 C)$.

A biomassa observada nos primeiros $10 \mathrm{~cm}$ da linha $A$ é baixa quando comparada à biomassa nas alturas de 10 a 20 e 20 a 30 $\mathrm{cm}$, apesar desta ser a região com a maior freqüência de alagamento. Este fato pode ser conseqüência de um maior estresse que as algas podem estar sofrendo devido à intensa deposição de sedimentos, que restringe a absorção de luz e as trocas gasosas e facilita o epifitismo por diatomáceas bentônicas. Já na região superior do tronco (acima de $40 \mathrm{~cm}$ de altura), a freqüência de alagamento é muito baixa, e apenas as algas mais resistentes à dessecação, principalmente Bostrychia radicans, conseguem apresentar valores representativos de biomassa (Figura 4A). Com relação às linhas $\mathrm{B}$ e $\mathrm{C}$, a biomassa decresce acima dos primeiros $10 \mathrm{~cm}$, devido à baixa freqüência de alagamento, uma vez que esta porção do manguezal está numa região com nível topográfico mais elevado.

Apesar da elevada variabilidade vertical e horizontal na biomassa e na comunidade das macroalgas no manguezal estudado, não há variações temporais bem definidas na biomassa da linha $A$, apesar de haver uma tendência para valores mais baixos no final do verão e início do outono (figura 3B). Mesmo observando-se cada altura da linha A separadamente não é possível evidenciar um padrão sazonal definido (figura 4A).

Com relação à composição da comunidade de algas, há aparentemente uma substituição de Bostrychia calliptera por Bostrychia pinnata do verão para o inverno, em todas as alturas da linha A (figura 4A). Esta tendência não é tão evidente nas linhas $B$ e $C$ (figura $4 \mathrm{~B}$ e 4C), e ainda deve ser vista com ressalvas, devido às dificuldades taxonômicas na separação destas duas espécies. 


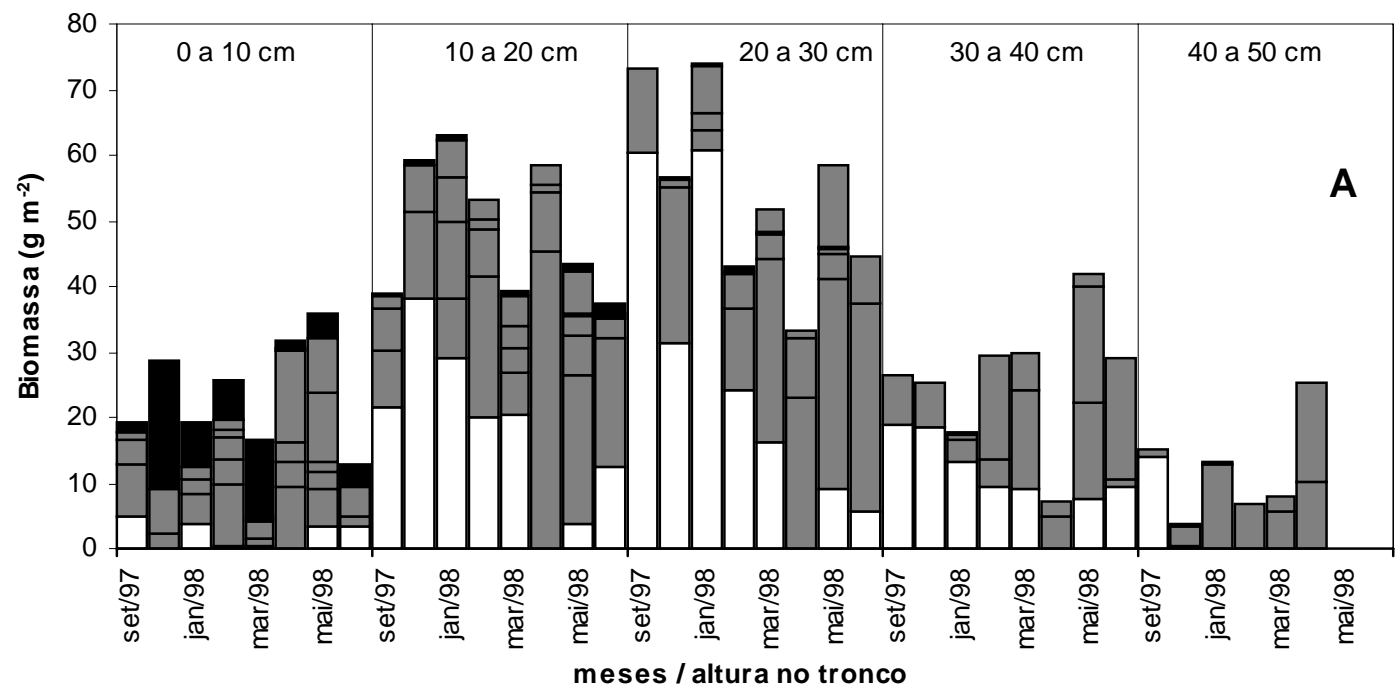

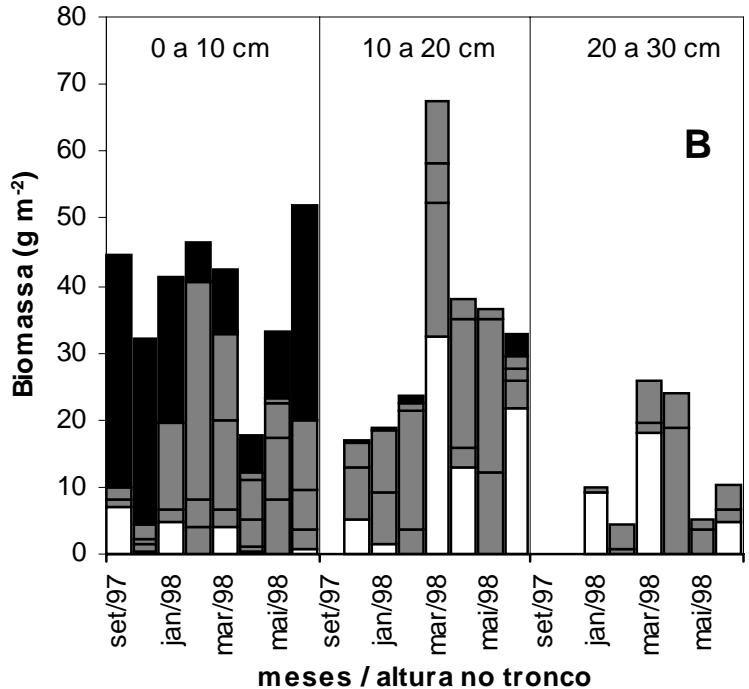
$\square$ B.calliptera
B.montagnei
$\square$ C.caespitosa

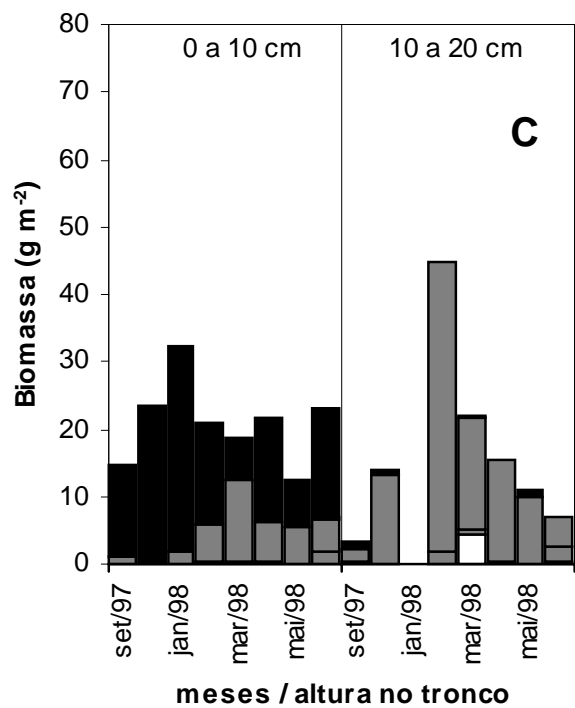

$\square$ B.radicans f. radicans

$\square$ C.leprieurii

Figura 4: Variação sazonal da biomassa de macroalgas de manguezal ( $\mathrm{g} \mathrm{m}-2$ de tronco), por espécie, para cada altura no tronco (com relação ao sedimento). A: Linha A (franja do manguezal); B: Linha B (porção intermediária do manguezal); C: Linha C (porção mais interna do manguezal). 
Na linha B, uma tendência sazonal pode ser observada com relação à biomassa média no tronco (figura $3 \mathrm{C}$ ), especialmente em função da variação da biomassa entre 10 e $20 \mathrm{~cm}$ (figura 4B). Nos primeiros $10 \mathrm{~cm}$ não há uma tendência de variação sazonal da biomassa, mas há uma forte tendência sazonal na dominância das algas. As algas verdes dão lugar às vermelhas do início para o final do verão, voltando a aumentar em percentual com a aproximação do inverno (figura 4B). Esta mesma tendência foi observada nos primeiros $10 \mathrm{~cm}$ da linha $C$ (figura $4 \mathrm{C}$ ).

Essas tendências de sazonalidade para as linha $B$ e $C$ podem estar relacionadas com a precipitação ou salinidade, que variaram ao longo do período. Como a linha $A$ apresenta maior freqüência de alagamento, as algas não sofreriam estresse por dessecação e por elevação da salinidade. Já nas linhas $B$ e $C$, como a freqüência de alagamento é baixa, as algas estão mais sujeitas a ao estresse por dessecação e por salinidade. Durante o período de maior pluviosidade, a precipitação melhora as condições para as algas das linhas $B$ e $C$, mas sua influência é menos evidente na linha $A$, que não sofre com tanta intensidade 0 estresse.

Entretanto, estes argumentos são apenas hipóteses, que precisam ser testadas com experimentos de campo e laboratório, e ainda com a observação da biomassa por de um período maior, que feche pelo menos um ciclo anual. Outro fator que pode estar interferindo na interpretação dos resultados, é o tempo de reposição da biomassa de algas (turnover rate). As algas são reconhecidamente organismos de grande dinâmica de biomassa, e amostragens mensais podem ser insuficientes para evidenciar uma variação de biomassa relacionada com parâmetros ambientais que apresentam uma grande variabilidade em curta escala temporal.

Mesmo para uma avaliação da biomassa instantânea de algas do manguezal como um todo, os resultados preliminares apresentados neste trabalho são ainda insuficientes. Estes resultados incluem apenas as algas presentes nos troncos e raízes da árvores. As algas presentes nos pneumatóforos ainda não foram consideradas. Como a densidade de pneumatóforos é bastante elevada no manguezal (de 100 a $400 \mathrm{~m}^{-2}$ ), e a biomassa de algas em cada pneumatóforo é bastante elevada (Cunha, dados não publicados) quanto este compartimento for computado, os valores de biomassa devem crescer consideravelmente, assim como a contribuição de cada espécie para a biomassa total deve mudar.

Embora a determinação da biomassa instantânea não permita boas estimativas de produção das macroalgas, apenas a biomassa instantânea de algas nos troncos ( $\mathrm{g} \mathrm{m}^{-2}$ de tronco) é similar à produção mensal de serrapilheira no mesmo manguezal (10 e $80 \mathrm{~g} \mathrm{~m}^{-2}$ mês $^{-1}$, Cunha, dados não publicados). Resultados preliminares sugerem que a alga Bostrychia montagnei apresenta taxas de crescimento variando entre 1 e $4 \%$ ao dia, e taxas fotossintéticas variando entre 0,8 e 1,2 $\mathrm{mg} \mathrm{O}_{2} \mathrm{~g}$ (peso fresco) $\mathrm{h}^{-1}$ (Cunha, dados não publicados).

Para tentar avaliar o quanto estaria sendo disponibilizado em termos de biomassa de macroalgas para o manguezal, experimentos complementares vem sendo desenvolvidos. Tais experimentos tentam avaliar o potencial produtivo destas algas e suas respostas aos principais fatores ambientais aos quais as mesmas estão sujeitas, entretanto os resultados ainda não estão disponíveis.

\section{AGRADECIMENTOS}

Agradecemos à Bióloga Maria Cristina M. Silva, à direção e aos mestres de embarcação do Joinville late Clube (JIC), à Fundação de Meio Ambiente de Joinville (FUNDEMA) e aos técnicos do CTTMar/ UNIVALI, pelo apoio técnico e logístico às saídas de campo. 


\section{REFERÊNCIAS}

Alveal, K. \& Romo, H. 1995. Estudios Zonacionales. pp. 611-641. In: Manual de Métodos Ficológicos. (ALVEAL, K.; FERRARIO, M.E.; OLIVEIRA, E.C. \& SAR, E. , eds). Concepcion, Chile: Universidad de Concepcion.

Amarasinghe, M.D. \& Balasubramaniam, S. 1992. Net primary productivity of two mangrove forest stands on the northwestern coast of Sri Lanka. Hydrobiologia. 247:37-47.

Ambler, J.W.; Alcala Herrera, J. \& Burke, R. 1993. Trophic roles of particle feeders and detritus in a mangrove island prop root ecosystem, 437-446. In: Ferrari, F. D.; Bradley, B. P. (eds). Ecology and Morphology of Copepods.

Braga, M. R. A.; FUJII, M. T.; Yokoya, N.S.; Eston, V.R.; Plastino, E. M. \& CordeiroMarino, M. 1990. Macroalgal repoductive patterns in mangroves of llha do Cardoso, SP, Brasil. In: Anais do II Simposio Sobre Ecossistemas da Costa Sul e Sudeste Brasileira. Estrutura, função e manejo. Vol. 1, pp. 314-326. ACIESP, SP, Brasil.

Brown, M. S. 1984. Mangrove leaf litter production and dynamics. In: Snedaker, S. C. \& Snedaker (eds.) The mangrove ecosystem: research methods. UNESCO.

Camargo, T. M. 1982. Comunidades naturais de raízes de mangue vermelho (Rhizophora mangle) e experimentos com substratos artificiais na região de Cananéia $\left(25^{\circ} \mathrm{S}\right)$ Brasil. Tese de Mestrado. Universidade de São Paulo, Brasil.

Cintron, G. \& Schaeffer-Novelli, Y. 1983. Roteiro para estudos dos recursos de marismas e manguezais. Relat. Int. Inst. Oceanogr. Univ. S. Paulo, 10:1-13.

Climerh/Epagri. 1997. Análise de dados climatológicos para a Região da Baía de Babitonda. Relatório.
Cordeiro-Marino, M. ; Braga, A.; Eston, V.R.; Fujii, M.T. \& Yokoya, N.S. 1992. Mangrove macroalgal communities of Latin America: The state of art and perspectives. In: Seeliger, U. (Ed.). coastal Plant Communities of Latin America.Academic Press, New York, pp. 51-64.

Cordeiro-Marino, M. 1978. Rodofíceas bentônicas marinhas do Estado de Santa Catarina. Richkia, São Paulo, 7:1-243.

Cunha, S.R.; Silva, M.C.M.; Nascimento, J. \& Lima, G.B. 1997. Caracterização estrutural dos manguezais do Canal de São Francisco do Sul e do Canal do Linguado, Baía da Babitonga, SC. X Semana Nacional de Oceanografia, Itajaí, SC, outubro de 1997.

Day Jr., J. W.; Hall, C. A.; Kemp, W. M. \& Yáñez-Arancibia, A. 1989. Estuarine Ecology. John Wiley \& Sons, New York, $558 \mathrm{pp}$.

Eston, V.R.; Rosario, M.; Braga, A.; CordeiroMarino, M.; Fujii, M.T. \& Yokoya, N.S. 1992. Macroalgal colonization patterns on artificial substrates inside southeastern Brazilian mangroves. Aquat Bot, 45:315325.

Eston, V.R.; Yokoya, N.S.; Fujii, M.T.; Braga, A.; Plastino, E.M. \& Cordeiro-Marino, M. 1991. Mangrove macroalgae in southeastern Brazil: spatial and temporal patterns. Rev. Bras. Biol., 51(4):829-837.

Flores-Verdugo, F.J., Day Jr., J.W., \& BriseñoDueñas, R.1987. Structure, litter fall, decomposition, and detritus dynamics of mangroves in a Mexican coastal lagoon with an ephemeral inlet. Mar.Ecol.Prog.Ser. 35:83-90.

Hadlich, R.M. \& Bouzon, Z.L. 1985. Contribuição ao levantamento taxonômico das algas marinhas bentônicas do mangue do itacorubi - Florianópolis - Santa Catarina - Brasil. II - Rhodophyta. Insula, 15:89-116. Hadlich, R.M. 1984. Contribuição ao levantamento taxonômico das algas marinhas bentônicas do mangue do Itacorubi - 
Florianópolis - Ilha de Santa Catarina Brasil. I - Chlorophyta.

Hanisak, M.D.1993. Nitrogen release from decomposing seaweeds: species and temperature effects. Journal of Applied Phycology, 5:175-181.

Herz, R. 1991. Manguezais do Brasil. Univervidade de São Paulo.

Joly, A.. B. 1954. The genus Bostrychia Montagne, 1838 in Southern Brazil. Taxonomic and ecological data. Bol. Fac. Fil. Cienc. Letr. Univ. São Paulo, 209, Botânica 13:7-21.

Joly, A.. B. 1957. Contribuição ao conhecimento da flora ficológica marinha da baía de Santos e arredores. Bol. Fac. Fil. Cienc. Letr. Univ. São Paulo, 217, Botânica 14:1196.

Karsten, U., Koch, S., West, J. A. \& Kirst, G. O. 1994. The intertidal red alga Bostrychia simpliciuscula Harvey ex J. Agardh from a mangrove swamp in Singapore: Acclimation to light and salinity. Aquatic Botany 48, 313-323.

King, R.J. \& Puttock,D.F. 1989. Morphology and taxonomy of Bostrychia and Stictosiphonia (Rhodomelaceae/ Rhodophyta). Australian Systematic Botany 2:1-73.

Lee, S. Y. 1995. Mangrove outwelling: a reveiw. Hydrobiologia, (295): 203-212.

Mackey, A.P. AND Smail, G. 1995. Spatial and temporal variation in litter fall of Avicennia marina (Forssk.) Vierh. in the Brisbane River, Queensland, Australia. Aquat.Bot. 52:133-142.

Mann, K. 1982. Ecology of coastal waters: a system approach. University of California Press. Verkeley, CA. 322 pp.

Michell, G. J.; Monteiro, D. F. \& Medina, R. S. 1974. Observações ficológicas no manguezal de piedade. Leandra 4/5:137143.

Miranda, P. T. C. 1986. Composição e distribuição das macroalgas bentônicas no manguezal do Rio Ceará (Estado do Ceará) Brasil. Tese de Mestrado. Universi- dade Federal de Pernambuco, Recife, Brasil.

Narasimha Rao, G.M. 1995. Seasonal growth, biomass and reproductive behavior of three species of red algae in Godavari Estuary, India. J.Phycol. 31:209-214, 1995.

Oliveira, E. C. 1969. Algas marinhas do Sul do Estado do Espírito Santo (Brasil). I. Ceramiales. Bol. Fac. Fil. Cienc. Letr. Univ. São Paulo, Botânica 26:1-277

Oliveira, E. C. 1984. Brazilian mangal vegetation with special emphasis on the seaweeds, p. 55-65. In: Por, F.D. \& Dor, I. (eds), Hydrobiology of the Mangal. Dr. W. Junk Plublishers, The Hague.

Paula, E. J.; Ugadim, Y. \& Kanagawa, A. I. 1989. Macroalgas de manguezais da llha de Maracá, Estado do Amapá, Brasil. Insula, Florianópolis, Suplem., 19:95-144.

Pedrini, A. G. 1980. Algas marinhas bentônicas da Baía de Sepetiba e arredores (Rio de Janeiro). Tese de mestrado. Universidade Federal do Rio de Janeiro.

Post, E. 1968. Zur Verbreitungs-Okologie des Bostrychietum . Hydrobiologia, 31:241316.

Rodriguez, C. \& Stoner, A.W. 1990. The epiphyte community of mangrove roots in a tropical estuary: Distribution and biomass. Aquatic Botany, 36:117-126.

SEPLAN-SC. 1988. Números de Santa Catarina. Secretaria de estado de Planejamento e Coordenação Geral. Florianópolis, SC. 491 pp.

Shirata, M.T. 1993. Algas marinhas bentônicas do manguezal de Guaraqueçaba, Município de Guaraqueçaba, Paraná, Brasil. Estudos de Biologia PUC-PR, 03(35):115.

Steinke, T.D. \& Naidoo, Y. 1990. Biomass of algae epiphytic on pneumatophores of the mangrove, Avicennia marina, in the St. Lucia estuary. S.Afr.J.Bot.S.Afr. Tydskr.Plantkd. 56(2):226-232. 
Tenore, K.. R. 1977. Growth of Capitella capitata cultures on various levels of detritus derived from different sources. Limnol. Oceanogr., 22:936-941.

Tovilla-Hernandez, C.T. \& Gonzales-Angelito, E. G. 1994. Produccion de hojarasca del manglar en tres sistemas lagunares del Golfo de Mexico y el Pacifico. Serie Grandes Temas de la Hidrobiologia: Los Sistemas Litorales - UAMI - UNAM. 2:87-103.

Tsuda, R.T. \& Abbott, I.A. 1985. Collection, handling, preservation and logistics, p. 6786. In: Littler, M.M. \& Littler, D.S. (eds), Handbook of Phycological Methods Ecological Field Methods: Macroalgae. Cambridge: Cambridge University Press.

Turner, R. E, \& Boesch, D. F. 1988. Aquatic animal production and wetland relationships: insights gleaned following wetland loss or gains, p. 25-39. In: Hook,
D.D. (Ed.), The ecology and management of wetlands. Croom Helm Ltd. Kent.

Turner, R. E. 1985. Coastal fisheries, agriculture and management in Indonesia: case studies for the future, p.373-440. In: Clark, J. R. (Ed.) Coastal resources management: development case studies. Research Planning Institute, Inc. Columbia, SC.

Vonshak, A. \& Maske, H. 1980. Algas: técnicas de crescimento e produção de biomassa, p.131-154. In: Coombs, J. \& Hall, D. O. (Eds.), Técnicas de Bioprodutividade e Fotossíntese. 